F. S. Cater, Department of Mathematics, Portland State University, Portland, Oregon 97207, USA

\title{
NOTE ON THE OUTER MEASURES OF IMAGES OF SETS
}

\begin{abstract}
Let $f$ be a real function on $\mathbb{R}$, let $\left\{I_{v}\right\}$ be a family of intervals covering a set $E$ such that $m\left(E \cap I_{v}\right) \geq m\left(f\left(E \cap I_{v}\right)\right)$ for each $I_{v}$. We prove that $m(f(E)) \leq 2 \cdot m(E)$. No coefficient smaller than 2 will suffice here in general.
\end{abstract}

This note concerns the following well-known [HS] result.

Proposition. Let $f$ be a real valued function on the line $\mathbb{R}$, differentiable at each point of a set $E \subset \mathbb{R}$, such that $\left|f^{\prime}(x)\right| \leq 1$ for each $x \in E$. Then $m(f(E)) \leq m(E)$, where $m$ denotes Lebesgue outer measure.

The usual proofs [HS] employ appropriate Vitali coverings of $E$ or of $f(E)$. We wonder if the conclusion holds when we dispense with the derivative and just let $E$ be covered by a family of intervals $\{I\}$ such that $m(E \cap I) \geq$ $m(f(E \cap I))$ for each $I$ in the family. That $m(E) \geq m(f(E))$ need not hold is shown in Proposition 1. Indeed $m(f(E))$ could be almost twice $m(E)$.

Theorem 1. Let $f$ be a real valued function on $\mathbb{R}$, let $E$ be a subset of $\mathbb{R}$ and let $\left\{I_{v}\right\}$ be a family of intervals covering $E$. Let $m\left(I_{v}\right) \geq m\left(f\left(E \cap I_{v}\right)\right)$ for each $I_{v}$. Then $m(f(E)) \leq 2 m\left(\cup_{v} I_{v}\right)$. If moreover $m\left(E \cap I_{v}\right) \geq m\left(f\left(E \cap I_{v}\right)\right)$ for each $I_{v}$, then $m(f(E)) \leq 2 \cdot m(E)$.

Proof. Let $U=\cup_{v}$ (interior $\left.I_{v}\right)$. Every point in $E \backslash U$ must be an endpoint of a component of $U$. Thus $E \backslash U$ is at most a countable set. Without loss of generality we can (and do) assume that $E \subset U$ and each $I_{v}$ is an open interval. By the Lindelöf Theorem [C], there are countably many intervals $I_{1}, I_{2}, I_{3}, \ldots$ such that $U=\cup_{j=1}^{\infty} I_{j}$.

Let $k$ be a real number such that $k<m(f(E))$. Choose an index $N$ such that

$$
m\left(f\left(E \cap \cup_{j=1}^{N} I_{j}\right)\right) \geq k .
$$

Key Words: coverings, Lebesgue outer measure

Mathematical Reviews subject classification: 28A12

Received by the editors October 31, 2000 
We consider each subfamily of $X=\left\{I_{1}, I_{2}, \ldots, I_{N}\right\}$ for which the union of its intervals equals $I_{1} \cup I_{2} \cup \ldots \cup I_{N}$. Let $\left\{K_{1}, K_{2}, \ldots, K_{t}\right\}$ be such a subfamily with a minimum number of intervals. Then no $K_{i}$ can be a subset of $\cup_{j \neq i} K_{j}$; otherwise we could delete $K_{i}$. Thus no two $K_{i}$ can have the same left endpoint. Say the $K_{i}$ are $\left(a_{1}, b_{1}\right),\left(a_{2}, b_{2}\right), \ldots,\left(a_{t}, b_{t}\right)$, where $a_{1}<a_{2}<\ldots<a_{t}$. Now $\left(a_{1}, b_{1}\right)$ cannot meet $\left(a_{j}, b_{j}\right)$ for any $j>2$; for if it did, either $\left(a_{j}, b_{j}\right) \subset\left(a_{2}, b_{2}\right)$ where $b_{j} \leq b_{2}$, or $\left(a_{2}, b_{2}\right) \subset\left(a_{1}, b_{1}\right) \cup\left(a_{j}, b_{j}\right)$ where $b_{j}>b_{2}$. It follows likewise that the intervals $\left(a_{1}, b_{1}\right),\left(a_{3}, b_{3}\right),\left(a_{5}, b_{5}\right), \ldots$ are mutually disjoint, and the intervals $\left(a_{2}, b_{2}\right),\left(a_{4}, b_{4}\right),\left(a_{6}, b_{6}\right), \ldots$ are mutually disjoint. But

$$
\begin{aligned}
m\left(\cup_{v} I_{v}\right) \geq & m\left(\left(a_{1}, b_{1}\right)\right)+m\left(\left(a_{3}, b_{3}\right)\right)+m\left(\left(a_{5}, b_{5}\right)\right)+\ldots \\
\geq & m\left(f\left(E \cap\left(a_{1}, b_{1}\right)\right)\right)+m\left(f\left(E \cap\left(a_{3}, b_{3}\right)\right)\right) \\
& +m\left(f\left(E \cap\left(a_{5}, b_{5}\right)\right)\right)+\ldots
\end{aligned}
$$

and

$$
\begin{aligned}
m\left(\cup_{v} I_{v}\right) \geq & m\left(\left(a_{2}, b_{2}\right)\right)+m\left(\left(a_{4}, b_{4}\right)\right)+m\left(\left(a_{6}, b_{6}\right)\right)+\ldots \\
\geq & m\left(f\left(E \cap\left(a_{2}, b_{2}\right)\right)\right)+m\left(f\left(E \cap\left(a_{4}, b_{4}\right)\right)\right) \\
& +m\left(f\left(E \cap\left(a_{6}, b_{6}\right)\right)\right)+\ldots
\end{aligned}
$$

We add (2) and (3) and obtain

$$
\begin{aligned}
2 \cdot m\left(\cup_{v} I_{v}\right) \geq & m\left(f\left(E \cap\left(a_{1}, b_{1}\right)\right)\right)+m\left(f\left(E \cap\left(a_{2}, b_{2}\right)\right)\right) \\
& +\ldots+m\left(f\left(E \cap\left(a_{t}, b_{t}\right)\right)\right) \\
\geq & m\left(f\left(E \cap \cup_{j=1}^{t}\left(a_{j}, b_{j}\right)\right)\right) .
\end{aligned}
$$

But because $\cup_{j=1}^{t}\left(a_{j}, b_{j}\right)=\cup_{i=1}^{N} I_{i}$

$$
m\left(f\left(E \cap \cup_{j=1}^{t}\left(a_{j}, b_{j}\right)\right)\right)=m\left(f\left(E \cap \cup_{i=1}^{N} I_{i}\right)\right) .
$$

From (1), (4) and (5) we obtain $2 \cdot m\left(\cup_{v} I_{v}\right) \geq k$. Because $k$ was arbitrary, we have $2 \cdot m\left(\cup_{v} I_{v}\right) \geq m(f(E))$.

For the second conclusion in Theorem 1 , replace $\left(a_{i}, b_{i}\right)$ with $E \cap\left(a_{i}, b_{i}\right)$ in (2), (3) and (4).

We now show that 2 is the smallest coefficient we can use in Theorem 1 . 
Proposition 1. For each number $d<2$, there is a continuous piecewise linear function $F_{d}$ on $\mathbb{R}$ and intervals $J_{d 1}, J_{d 2}$ in $\mathbb{R}$ for which

$$
m\left(J_{d 1}\right)=m\left(J_{d 2}\right)=m\left(F_{d}\left(J_{d 1}\right)\right)=m\left(F_{d}\left(J_{d 2}\right)\right),
$$

and

$$
m\left(F_{d}\left(J_{d 1} \cup J_{d 2}\right)\right)>d \cdot m\left(J_{d 1} \cup J_{d 2}\right) .
$$

Proof. Fix a real number $p$ for which $0<p<\frac{1}{4}$. In the plane $\mathbb{R}^{2}$, draw the segments from $(-\infty, 0)$ to $(0,0)$, from $(0,0)$ to $(p, 1)$, from $(p, 1)$ to $(1-p, 1)$, from $(1-p, 1)$ to $(1,2)$, and from $(1,2)$ to $(\infty, 2)$. Let $f_{p}$ be the real function on $\mathbb{R}$ whose graph is the broken line just constructed. From this graph we infer that $f_{p}\left(\frac{p^{2}}{1-p}\right)=\frac{p}{1-p}, f_{p}\left(\frac{1-p-p^{2}}{1-p}\right)=\frac{2-3 p}{1-p}$ and $f_{p}(p)=f_{p}(1-p)=1$. Let $I_{1}$ be the interval on the $x$-axis with endpoints $x=\frac{p^{2}}{1-p}$ and $x=1-p$. Let $I_{2}$ be the interval on the $x$-axis with endpoints $x=p$ and $\frac{1-p-p^{2}}{1-p}$. It follows that the interval $f_{p}\left(I_{1}\right)$ on the $y$-axis has endpoints $y=\frac{p}{1-p}$ and $y=1$; the interval $f_{p}\left(I_{2}\right)$ on the $y$-axis has endpoints $y=1$ and $y=\frac{2-3 p}{1-p}$. Direct computations give

$$
\begin{gathered}
m\left(I_{1}\right)=m\left(I_{2}\right)=m\left(f_{p}\left(I_{1}\right)\right)=m\left(f_{p}\left(I_{2}\right)\right)=\frac{1-2 p}{1-p}, \\
m\left(I_{1} \cup I_{2}\right)=\frac{1-p-2 p^{2}}{1-p} \text { and } m\left(f_{p}\left(I_{1} \cup I_{2}\right)\right)=\frac{2-4 p}{1-p} .
\end{gathered}
$$

It follows that $\frac{m\left(f_{p}\left(I_{1} \cup I_{2}\right)\right)}{m\left(I_{1} \cup I_{2}\right)}=\frac{2-4 p}{1-p-2 p^{2}}$. Clearly, for any positive number $d<2$, there is a positive number $p<\frac{1}{4}$ (depending on $d$ ), for which

$$
m\left(f_{p}\left(I_{1} \cup I_{2}\right)\right)>d \cdot m\left(I_{1} \cup I_{2}\right) .
$$

We infer from this the conclusion of Proposition 1.

We now list some consequences of our Theorem 1.

Corollary 1. Let $f \in \mathbb{R}^{\mathbb{R}}$ and let $\left\{I_{v}\right\}$ be a family of intervals such that (diameter $\left.f\left(I_{v}\right)\right) \leq\left(\operatorname{diameter} I_{v}\right)$ for each $I_{v}$. Then $m\left(f\left(\cup_{v} I_{v}\right)\right) \leq 2 \cdot m\left(\cup_{v} I_{v}\right)$.

Proof. Note that $m\left(f\left(I_{v}\right)\right) \leq\left(\right.$ diameter $\left.f\left(I_{v}\right)\right)$. Put $E=\cup_{v} I_{v}$ in Theorem 1 .

Corollary 2. Let $f$ be absolutely continuous on $\mathbb{R}$ and let $\left\{I_{v}\right\}$ be a family of intervals such that $\cup_{v} I_{v} \subset[0,1], m\left(\cup_{v} I_{v}\right)=1$ and $m\left(f\left(I_{v}\right)\right) \leq m\left(I_{v}\right)$ for each $I_{v}$. Then $\max f[0,1]-\min f[0,1] \leq 2$. 
Proof. Now $\cup_{v} I_{v}$ is evidently measurable and $m\left([0,1] \backslash\left(\cup_{v} I_{v}\right)\right)=0$. Because $f$ is absolutely continuous we have $m\left(f\left([0,1] \backslash\left(\cup_{v} I_{v}\right)\right)\right)=0$. Apply Theorem 1 with $E=\cup_{v} I_{v}$.

Corollary 3. Let $f$ be continuous on $\mathbb{R}$ and let $\left\{I_{v}\right\}$ be a family of intervals such that $\cup_{v} I_{v} \subset[0,1]$, the set $[0,1] \backslash\left(\cup_{v} I_{v}\right)$ is a countable set, and $m\left(f\left(I_{v}\right)\right) \leq$ $m\left(I_{v}\right)$ for each $I_{v}$. Then $\max f[0,1]-\min f[0,1] \leq 2$.

Proof. Use a scheme much like the proof of Corollary 2.

Corollary 4. Let $f$ be nondecreasing and let $\left\{\left(a_{v}, b_{v}\right)\right\}$ be a family of intervals such that $f\left(b_{v}\right)-f\left(a_{v}\right) \leq b_{v}-a_{v}$ for each interval $\left(a_{v}, b_{v}\right)$. Then

$$
m\left(f\left(\cup_{v}\left(a_{v}, b_{v}\right)\right)\right) \leq 2 \cdot m\left(\cup_{v}\left(a_{v} \cdot b_{v}\right)\right) .
$$

Proof. Corollary 1.

Corollary 5. Let $f$ be a continuous strictly increasing function on $\mathbb{R}$ and let $\left\{\left(a_{v}, b_{v}\right)\right\}$ be a family of intervals such that $f\left(b_{v}\right)-f\left(a_{v}\right) \geq b_{v}-a_{v}$ for each $\left(a_{v}, b_{v}\right)$. Then $m\left(f\left(\cup_{v}\left(a_{v}, b_{v}\right)\right)\right) \geq \frac{m\left(\cup_{v}\left(a_{v}, b_{v}\right)\right)}{2}$.

Proof. Let $g$ be the inverse function of $f$ on $f(\mathbb{R})$. Apply Corollary 4 to $g$ and the family of intervals $\left\{f\left(a_{v}\right), f\left(b_{v}\right)\right\}$.

In the proof of Theorem 1 we should not expect that there necessarily exists a subfamily of mutually nonoverlapping intervals $I_{1}, I_{2}, I_{3}, \ldots$ with $\sum_{j} m\left(I_{j}\right) \geq \frac{m\left(\cup_{v} I_{v}\right)}{2}$. Let, for example, $J_{n}=\left(-5^{-n}, \sum_{i=1}^{n} 2^{-i}\right)$ for each positive integer $n$, and $E=\left(\cup_{n=1}^{\infty} J_{n}\right) \cup\left(\cup_{n=1}^{\infty}\left(-J_{n}\right)\right)$. Then each subfamily of mutually nonoverlapping intervals is a singleton family, but the length of no interval here is as large as $\frac{m(E)}{2}$.

\section{References}

[C] H. Cullen, Introduction to General Topology, D. C. Heath, Boston, 1968 (Theorem 18.15).

[HS] E. Hewitt and K, Stromberg, Real and Abstract Analysis, Springer- Verlag, New York, 1965 (Exercises (17.25), (17.26), (17.27)). 